\title{
An Utstein-based model score to predict survival to hospital admission: The UB-ROSC score
}

\author{
Enrico Baldi ${ }^{\mathrm{a}, \mathrm{b}, *, 1}$, Maria Luce Caputo ${ }^{\mathrm{a}, \mathrm{c}, 1}$, Simone Savastano $^{\mathrm{d}}$, Roman Burkart ${ }^{\mathrm{e}}$, Catherine Klersy ${ }^{\mathrm{f}}$, \\ Claudio Benvenuti ${ }^{\mathrm{e}}$, Vito Sgromo ${ }^{\mathrm{g}}$, Alessandra Palo ${ }^{\mathrm{g}}$, Roberto Cianella ${ }^{\mathrm{h}}$, Elisa Cacciatore ${ }^{\mathrm{a}}$, \\ Luigi Oltrona Visconti ${ }^{\mathrm{d}}$, Gaetano Maria De Ferrari ${ }^{\mathrm{a}, \mathrm{b}}$, Angelo Auricchio ${ }^{\mathrm{c}}$ \\ a Department of Molecular Medicine, Section of Cardiology, University of Pavia, Pavia, Italy \\ b Cardiac Intensive Care Unit, Arrhythmia and Electrophysiology and Experimental Cardiology, Fondazione IRCCS Policlinico San Matteo, Pavia, Italy \\ c Cardiocentro Ticino, Lugano, Switzerland \\ d Division of Cardiology, Fondazione IRCCS Policlinico San Matteo, Pavia, Italy \\ e Fondazione TicinoCuore, Lugano, Switzerland \\ f Service of Clinical Epidemiology and Biometry, Fondazione IRCCS Policlinico San Matteo, Pavia, Italy \\ ${ }^{g}$ AREU Azienda Regionale Emergenza Urgenza, AAT Pavia c/o Fondazione IRCCS Policlinico San Matteo, Pavia, Italy \\ ${ }^{\mathrm{h}}$ Federazione Cantonale Ticinese Servizi Autoambulanze, Lugano, Switzerland
}

\section{A R T I C L E I N F O}

\section{Article history:}

Received 30 December 2019

Accepted 13 January 2020

Available online xxxx

\section{Keywords:}

Out of hospital cardiac arrest

Survival

Prediction

Return of spontaneous circulation

Score

\begin{abstract}
A B S T R A C T
Aims: To develop and validate a multi-parametric practical score to predict the probability of survival to hospital admission of an out-of-hospital cardiac arrest (OHCA) victim by using Utstein Style-based variables.

Methods: All consecutive OHCA cases occurring from 2015 to 2017 in two regions, Pavia Province (Italy) and Canton Ticino (Switzerland) were included. We used random effect logistic regression to model survival to hospital admission after an OHCA. We computed the model area under the ROC curve (AUC ROC) for discrimination and we performed both internal and external validation by considering all OHCAs occurring in the aforementioned regions in 2018. The Utstein-Based ROSC (UB-ROSC) score was derived by using the coefficients estimated in the regression model. The score value was obtained adding the pertinent score components calculated for each variable. The score was then plotted against the probability of survival to hospital admission.

Results: 1962 OHCAs were included (62\% male, mean age $73 \pm 16$ years). Age, aetiology, location, witnessed OHCA, bystander CPR, EMS arrival time and shockable rhythm were independently associated with survival to hospital admission. The model showed excellent discrimination (AUC $0.83,95 \% \mathrm{CI} 0.81-0.85$ ) for predicting survival to hospital admission, also at internal cross-validation (AUC $0.82,95 \% \mathrm{Cl} 0.80-0.84$ ). The model maintained good discrimination after external validation by using the 2018 OHCA cohort (AUC $0.77,95 \% \mathrm{Cl} 0.74-0.80$ ). Conclusions: UB-ROSC score is a novel score that predicts the probability of survival to hospital admission of an OHCA victim. UB-ROSC shall help in setting realistic expectations about sustained ROSC achievement during resuscitation manoeuvres.
\end{abstract}

(c) 2020 The Authors. Published by Elsevier B.V. This is an open access article under the CC BY-NC-ND license (http:// creativecommons.org/licenses/by-nc-nd/4.0/).

\section{Introduction}

Out-of-hospital cardiac arrest (OHCA) is the most frequent cause of death in the industrialized world [1,2]. OHCA outcome significantly varies across different countries [3]; it is influenced by numerous underlying independent variables related to the patient's characteristics, e.g. age, comorbidity, and the circumstances in which the event occurred (aetiology, witnessed or not, public or private location, etc.) [4] as well

\footnotetext{
* Corresponding author at: c/o Fondazione IRCCS Policlinico San Matteo, Viale Golgi 19, 27100 Pavia, Italy.

E-mail address: enrico.baldi@unipv.it (E. Baldi).

1 These authors contributed equally.
}

as by modifiable factors, including bystander cardiopulmonary resuscitation (CPR), use of automated external defibrillators (AED) and the intervention time of emergency medical services (EMS) [5,6]. Similarly to survival, also the probability of return of spontaneous circulation (ROSC) significantly varies across geographies [3]. Prompt initiation of CPR manoeuvres after OHCA has an immediate impact on both ROSC achievement and outcome. ROSC in field can be temporarily or permanently achieved, bearing in mind that the most important one in OHCA management is a sustained ROSC. The development of a userfriendly and generally applicable tool to predict the resuscitation success, indicated by a sustained ROSC leading to hospital admission after arrival of the EMS team, would be of major clinical relevance. Such a score - made up off different independent variables which are readily 
available to the EMS team - could support the decision for aggressive therapy, provide medical personnel and family members with reasonable expectation of immediate outcome as well as improve general termination-of-resuscitation rules beyond those currently available [7-11].

Recommended guidelines for uniform OHCA data reporting have been developed as the Utstein Style [12,13]; thus, a basis for comparison has been created by providing clear data definitions. Utstein Style data reporting already contains key prognostic variables associated with survival or death following cardiac arrest, but to our knowledge, there has been no effort to develop a predictive score of sustained ROSC using these variables.

Therefore, the aim of our study was to develop and validate a multiparametric score to predict the probability of sustained ROSC and survival to hospital admission of OHCA victims by using the Utstein templates for patient data collection.

\section{Methods}

\subsection{Population}

All consecutive patients suffering OHCAs of any aetiology between January 1st, 2015 and December 31st, 2017 in the province of Pavia, Northern Italy, and in the Canton of Ticino, Southern Switzerland, were included in the study. Patients declared dead before ambulance arrival, with a "do not resuscitate" order, and/or with incomplete or unknown data were excluded from further analysis. A second cohort of consecutive patients from the same centers admitted between January 1st, 2018 and December 31st, 2018 was also enrolled for external validation using the same inclusion/exclusion criteria.

\subsection{Study design and setting}

This is a prospectively designed development of a multiparametric practical score to predict the probability of survival to hospital admission of OHCA victims by using Utstein templates for patient data collection in 2 large regional registries. Both the province of Pavia and the Canton of Ticino have a prospectively designed registry of cardiac arrest, i.e. the Cardiac Arrest Registry of the Province of Pavia (Pavia CARe), and the Ticino Region Cardiac Arrest Registry (TiReCa). Both registries follow the Utstein recommendations for data collection [12,13], as previously described $[14,15]$, periodically reviewed for quality assessment by an internal commission, and were approved by the local ethical committees.

\subsection{EMS and resuscitation network in the province of Pavia}

The province of Pavia is a region of $2965 \mathrm{~km}^{2}$ with several rural and a few urban areas with a total population of 548,722 inhabitants (as per December 31st, 2014). A national emergency telephone number, 112, is connected to the regional EMS dispatching centre. The local EMS dispatcher coordinates 33 ambulances staffed with Basic Life Support Defibrillation (BLS-D) trained personnel, and 5 ALS-trained staffed vehicles. In case of suspected OHCA, the EMS dispatcher activates one or two emergency vehicles of which at least one with a physician on board and assists the calling bystander during chest compressions (telephone CPR).

\subsection{EMS and resuscitation network in the Canton of Ticino}

The Swiss Canton of Ticino has a population of 350,363 inhabitants (as per December 31st, 2014). It covers a territory of $>2800 \mathrm{~km}^{2}$ in the southern part of Switzerland. The population is distributed among some cities (population ranging from 5000 to 70,000 inhabitants) and few hundreds of rural municipalities. A national emergency telephone number -144 , is connected to each of the six regional EMS dispatching centres operating in the Canton of Ticino, which in turn coordinate seven EMS services, all with ALS-trained staffed vehicles. When a cardiac arrest is suspected, a telephone assisted CPR is initiated until the arrival of an ambulance. The EMS dispatchers send the ambulance and, in parallel, alert the traditional first-responders represented by police officers and fire brigade, all trained in BLS-D and equipped with AEDs. If the OHCA condition is regarded as safe, the lay responders' network is also activated and automatically managed by a mobile application [16]. Their training includes the standard Swiss Resuscitation Council Basic Life Support (SRC BLS)/AED course for lay rescuers which complies with the recommendations of the European Resuscitation Council. Each EMS service individually collects data about OHCA interventions in the registry pursuant the Utstein methodology. An internal commission then periodically performs a quality assessment review of the data. None of 2 EMS systems have had or has rules for termination of resuscitation (TOR). The decision to terminate resuscitation is always at discretion of the pre-hospital team/physician until arrival at emergency room. However, patient's characteristics (e.g. age, comorbidities, etc.), OHCA circumstances (e.g. presence of witness, bystander CPR, etc.), and additional resuscitation elements (e.g. cardiac rhythm, end-tidal $\mathrm{CO}_{2}$ value before and during $\mathrm{CPR}$, duration of resuscitation effort, etc.) may guide a TOR decision.

\subsection{Definition of survival to hospital admission (sustained ROSC)}

The survival to hospital admission of the patient was defined as a patient in whom a ROSC was sustained until arrival at the emergency department and transfer of care to medical staff at the receiving hospital. This definition corresponds to the Utstein recommendations' core outcome - "Survived event" [13].

\subsection{Predictors identification for survival to hospital admission}

Age, aetiology, OHCA location site, witnessed OHCA, bystander CPR, time of EMS arrival and presenting rhythm are all Utstein variables readily available at EMS arrival being considered of prognostic relevance according to recent literature [13,17-23].

\subsection{Statistical analysis}

Each and every analysis was performed by using Stata 15.1 (StataCorp, College Station, TX, USA). Variables are given as mean and standard deviation (SD), or the median and 25th-75th percentiles if continuous, and with counts and percent if categorical. Comparisons were made by means of the Mann Whitney $U$ test or Fisher exact test. A random effect logistic regression, with a random effect for center, was used to develop the model for sustained ROSC. Odds ratios (OR) and their 95\% confidence intervals $(95 \% \mathrm{CI})$ were computed. The model area under the ROC curve (AUC ROC) was computed for discrimination. Model sensitivity (to identify survivors) and specificity (to identify non survivors) with predictive values for a probability of hospital survival $>50 \%$ was estimated, and model calibration was graphically assessed. A 10 -fold cross validation to obtain optimism corrected AUC-ROC was used (internal validation). The coefficients estimated from the model, multiplied by 10 and rounded to the closest integer were used to compute a prediction score; the score was then plotted against the probability of survival to hospital admission to provide an easy to use nomogram. The score was then applied to the validation cohort, and the area under the ROC curve $(95 \% \mathrm{CI})$ against survival to hospital admission was computed for external validation.

\section{Results}

Overall, 3186 patients suffered from an OHCA during the study period. Of these, 1109 (34.8\%) were declared dead before ambulance arrival or had a "do not resuscitate" order, 115 patients (3.6\%) had 
incomplete data thus, being excluded from any subsequent analysis. The demographic characteristics of the 1962 OHCA victims (1383 cases in the province of Pavia and 579 cases in the Canton of Ticino) representing the derivation cohort, and circumstances of the arrest are presented in Table 1. Although the population groups from the two centers differed statistically in most of the variables, some of them could be considered modestly different from the clinical standpoint. Indeed, the age was similar (Ticino: 71.1 yrs old vs Pavia: 73.4 yrs old, $p<0.001$ ) as well as time to EMS arrival (Ticino: $11.3 \mathrm{~min}$ vs. Pavia: $11.6 \mathrm{~min}$, $p=0.09$ ). On the other hand, there were more males in Ticino (66.6\%) compared to Pavia $(60.5 \%, p<0.01)$, OHCA was less frequently due to a cardiac cause in Ticino (77.9\%) vs Pavia $(92.8 \%, p<0.001)$ and occurred less frequently at home in the Ticino cohort (69.1\%) compared to the Pavia cohort $(79.6 \%, p<0.001)$. A non-shockable rhythm was more frequent in the Pavia cohort $(81.6 \%$ vs. $74.8 \%, p<0.001)$, most likely due to a less frequent bystander CPR ( $33.8 \%$ vs. $71.2 \%, p<0.001$ ) thus, resulting in a lower survival to hospital admission (16.9\% vs. $33.5 \%, p<0.001)$.

\subsection{Predictors of survival to hospital admission}

The multivariable logistic model for survival to hospital admission, including all the acknowledged prognostic factors from the literature, is summarized in Table 2. The model shows an AUC of $0.83(95 \% \mathrm{CI}$ 0.81-0.85; Fig. 1), and a good calibration (Fig. 1). Excellent discrimination was found at the internal validation (AUC $0.82,95 \% \mathrm{CI} 0.80-0.84$, after 10 -fold cross-validation). All variables were independently associated with survival to hospital admission. The coefficients derived from the model, and simplified, were used to calculate a prediction score, the proposed Utstein-based ROSC score (UB-ROSC), that can be easily computed by summing up the pertinent score components reported in Table 2, last column. It varies from -50 to +44 . The probabilities of survival to hospital admission (with $95 \% \mathrm{CI}$ ) are tabulated (left panel)

Table 1

Characteristics of the derivation and validation cohorts.

\begin{tabular}{|c|c|c|}
\hline Variable & $\begin{array}{l}\text { Derivation cohort } \\
\text { (year } \\
2015-2017 \text { ) }\end{array}$ & $\begin{array}{l}\text { Validation } \\
\text { cohort } \\
\text { (year 2018) }\end{array}$ \\
\hline Patients included & 1962 & 747 \\
\hline Male gender & $1223(62.3)$ & $442(59.2)$ \\
\hline Age, mean $\pm S D$ & $72.7 \pm 15.7$ & $73.7 \pm 16.1$ \\
\hline Age $\geq 80$ & $786(40.1)$ & $347(46.4)$ \\
\hline \multicolumn{3}{|l|}{ Aetiology } \\
\hline Cardiac & $1734(88.4)$ & $623(83.4)$ \\
\hline Trauma & $83(4.2)$ & $37(4.9)$ \\
\hline Drowning & $6(0.3)$ & $7(0.9)$ \\
\hline Respiratory & $91(4.6)$ & $52(7)$ \\
\hline Other non-cardiac & $48(2.5)$ & $28(3.8)$ \\
\hline \multicolumn{3}{|l|}{ Witness } \\
\hline None & $540(27.5)$ & $224(30)$ \\
\hline Bystander & $1137(58)$ & $414(55.4)$ \\
\hline EMS & $285(14.5)$ & 109 (14.6) \\
\hline \multicolumn{3}{|l|}{ Location } \\
\hline Home & $1501(76.5)$ & $556(74.4)$ \\
\hline Nursing home & $149(7.6)$ & $77(10.3)$ \\
\hline Work place & $26(1.3)$ & $9(1.2)$ \\
\hline School & $1(0.1)$ & $0(0)$ \\
\hline Street & $176(9)$ & $57(7.6)$ \\
\hline Public building & $72(3.6)$ & $35(4.7)$ \\
\hline Sport & 37 (1.9) & $13(1.8)$ \\
\hline \multicolumn{3}{|l|}{ Rhythm } \\
\hline Not Shockable & $1562(79.6)$ & $641(82.2)$ \\
\hline Shockable & $400(20.4)$ & $133(17.8)$ \\
\hline \multicolumn{3}{|l|}{ Bystander CPR } \\
\hline Yes & $879(44.8)$ & $327(43.8)$ \\
\hline No & $1083(55.2)$ & $420(56.2)$ \\
\hline $\begin{array}{l}\text { Time to EMS arrival, mean in minutes } \\
\text { (IQR) }\end{array}$ & $11.5(7.9-14)$ & $13.8(8-15)$ \\
\hline Survival to hospital admission & $428(21.8)$ & $197(26.4)$ \\
\hline
\end{tabular}

and plotted as a nomogram (right panel) in Fig. 2. A score of 0 corresponds to a $50 \%$ probability of survival to hospital admission. The calculation of two simulated cases (red \& green dots) is shown in the figure legend.

\subsection{External validation of the UB-ROSC score}

The UB-ROSC score was further validated in a cohort composed of 747 OHCAs patients enrolled in 2018, 537 in the Pavia registry and 210 in the Ticino registry (Table 1, validation cohort). In this external validation, the model maintained a discrimination value of 0.77 (95\% CI 0.74-0.80) for predicting survival to hospital admission as compared to the 0.82 obtained from the internal validation (see above).

\section{Discussion}

To the best of our knowledge, the Utstein-based ROSC (UB-ROSC) score, is the first available score predicting sustained ROSC and survival to hospital admission of patients after having suffered an OHCA. The UBROSC score shows excellent discrimination capability and already accounts for a random effect related to center, thus making it a score to be used in-field, and possibly supporting resuscitation-related decisions such as considering termination of resuscitation manoeuvres.

Resuscitation termination rules are still matter of debate. Previous studies suggested some resuscitation termination rules capable to identify patient without a chance of survival; however the generalisability of these rules has been significantly questioned [7-11,24,25]. Indeed, it is unlikely to be able to achieve a positive predictive value of $100 \%$, and even a small chance of survival shall not be a-priori neglected. However, it is important to emphasize that a low UB-ROSC score should never lead to withhold resuscitation, since a resuscitation attempt should be performed in all patients, even with a minimal probability of survival. The utility of UB-ROSC is instead to help in setting realistic expectations about the likelihood of achieving sustained ROSC during resuscitation manoeuvres. This knowledge is important for paramedics, rescue teams and even more so for family members. The awareness and understanding that the circumstances of OHCA and the demographic characteristics of the cardiac arrest victim play a significant role in the possibility of survival to hospital admission is particular relevant for acceptance of poor outcome by family members; despite that, it is particularly important to make every effort to save patients' lives.

The UB-ROSC score shares some important prognostic elements with the ACLS score proposed by Eisenberg et al. [26], and with the ROSC After Cardiac Arrest (RACA) score by Gräsner et al. [23], but is significantly different in the way it was designed to predict outcome at variance with the other two. All three scores have been developed to formulate a prediction of the probability of the patient surviving based on variables that are both predictive of survival and readily available at the scene of the emergency. Both the ACLS score and the UB-ROSC score are operational scores whereas the RACA score is not meant to be used as a practical score by the EMS personnel at arrival on the scene. The RACA score was on the other hand developed as an instrument to facilitate the comparison of different ROSC percentages in studies performed in different conditions and enrolling patients with different characteristics. Therefore, it is useful to enable comparison between different EMS systems, educational levels, technical equipment, and therapeutic interventions, but it cannot be used to help EMS personnel to assess survival probability of OHCA victims in a real-life scenario [23]. The ACLS was developed as a score predictive of outcome (specifically, discharge alive from hospital) following OHCA [26], whereas UB-ROSC is a score predictive of sustained ROSC and survival to hospital admission. Although the development and use of an practical score predicting OHCA patients' survival following hospital treatment would represent the best score possible; in daily practice it fails short because it includes the uncertainties and weakness of each ring of the so-called "chain-of-survival" [27]. Endpoints such as 'hospital discharge' 
Table 2

Multivariable logistic model for survival to hospital admission and score computation.

\begin{tabular}{|c|c|c|c|c|}
\hline Candidate predictors ${ }^{*}$ & Survival to hospital admission (\%) & OR $(95 \% \mathrm{CI})$ & $p$ & Score component \\
\hline \multicolumn{5}{|l|}{ Sex } \\
\hline Female & $141(19.1)$ & 1.00 & & 0 \\
\hline Male & $287(23.5)$ & $0.74(0.56-0.99)$ & 0.044 & -3 \\
\hline \multicolumn{5}{|l|}{ Age } \\
\hline$<80$ & $345(29.3)$ & 1.00 & & 0 \\
\hline$\geq 80$ & $83(10.5)$ & $0.39(0.29-0.54)$ & $<0.001$ & -9 \\
\hline Aetiology & & & $<0.001$ & \\
\hline Cardiac & 359 (20.7) & 1.00 & & 0 \\
\hline Trauma & $11(13.3)$ & $0.72(0.35-1.52)$ & 0.394 & -3 \\
\hline Drowning & $1(16.7)$ & $1.09(0.10-11.77)$ & 0.945 & 1 \\
\hline Respiratory & $46(50.5)$ & $6.72(4.03-11.20)$ & $<0.001$ & 19 \\
\hline Other non-cardiac & $11(22.9)$ & $0.95(0.44-2.05)$ & 0.906 & 0 \\
\hline \multicolumn{3}{|l|}{ Location } & 0.014 & \\
\hline At home & $292(19.5)$ & 1.00 & & 0 \\
\hline Nursing home & $16(10.7)$ & $0.51(0.27-0.95)$ & 0.035 & -7 \\
\hline Workplace & $12(46.2)$ & $1.90(0.76-4.77)$ & 0.173 & 6 \\
\hline School & $1(100)$ & 1.00 & & 0 \\
\hline Street & $54(30.7)$ & $1.50(0.96-2.35)$ & 0.075 & 4 \\
\hline Public building & $35(48.6)$ & $1.70(0.95-3.02)$ & 0.072 & 5 \\
\hline Sport & $18(48.7)$ & $2.00(0.91-4.42)$ & 0.087 & 7 \\
\hline \multicolumn{3}{|l|}{ Bystander and CPR } & $<0.001$ & \\
\hline No witnessed, no CPR & $30(9.6)$ & 1.0 & & 0 \\
\hline No witnessed, yes CPR & $31(13.7)$ & $0.59(0.32-1.09)$ & 0.091 & -5 \\
\hline Witnessed, no CPR & $66(13.2)$ & $1.19(0.72-1.97)$ & 0.502 & 2 \\
\hline Witnessed, yes CPR & $200(31.4)$ & $1.51(0.93-2.44)$ & 0.094 & 4 \\
\hline EMS witnessed & $101(35.4)$ & $3.61(2.18-5.96)$ & $<0.001$ & 13 \\
\hline \multicolumn{3}{|l|}{ Rhythm } & $<0.001$ & \\
\hline Not Shockable & $201(12.9)$ & 1.00 & & 0 \\
\hline Shockable & $227(56.8)$ & $8.18(6.08-11.02)$ & & 21 \\
\hline \multicolumn{3}{|l|}{ Time arrival EMS } & $<0.001$ & \\
\hline$\leq 10 \mathrm{~min}$ & $247(25.3)$ & 1.00 & & 0 \\
\hline $11-15 \mathrm{~min}$ & $130(19.9)$ & $0.69(0.52-0.92)$ & 0.012 & -4 \\
\hline$\geq 15 \min$ & $51(15.4)$ & $0.49(0.33-0.72)$ & $<0.001$ & -7 \\
\hline \multicolumn{2}{|l|}{ Constant } & $0.20(0.10-0.39)$ & & -16 \\
\hline \multicolumn{4}{|l|}{ Model performance } & Model statistics \\
\hline \multicolumn{4}{|l|}{ Model $p$} & $<0.001$ \\
\hline \multicolumn{4}{|l|}{ AUC ROC (95\%CI) } & $0.83(0.81-0.85)$ \\
\hline \multicolumn{4}{|l|}{ Model sensitivity } & $50.2(45.4-55.1)$ \\
\hline \multicolumn{4}{|l|}{ Model specificity } & $92.9(91.5-94.1)$ \\
\hline \multicolumn{4}{|c|}{ Model positive predictive value of a probability of survival > 50\% } & $66.4(60.9-71.5)$ \\
\hline \multicolumn{4}{|c|}{ Model negative predictive value of a probability of survival $>50 \%$} & $87(85.3-88.6)$ \\
\hline \multicolumn{4}{|c|}{10 -fold cross validation AUC ROC (95\%CI) } & $0.82(0.80-0.84)$ \\
\hline \multicolumn{4}{|c|}{ Validation in 2018 cohort } & $0.77(0.74-0.80)$ \\
\hline
\end{tabular}

* All variables were significantly associated with survival to hospital admission at the univariable analysis.
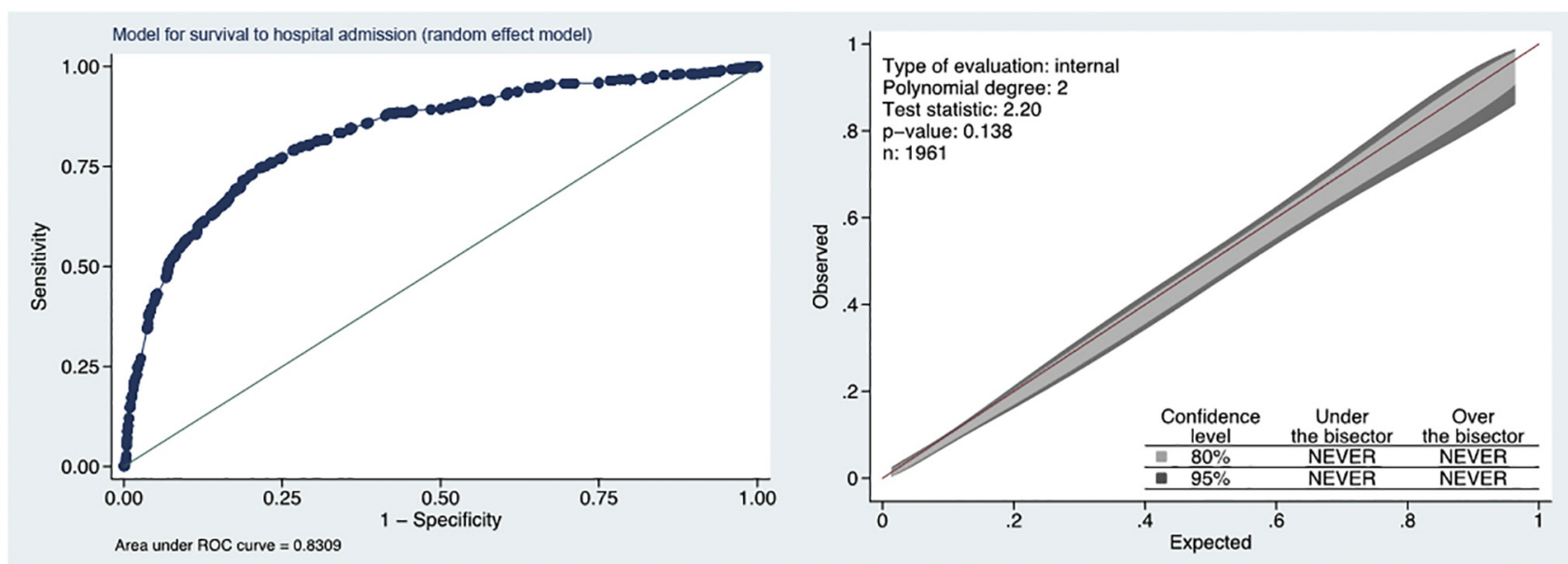

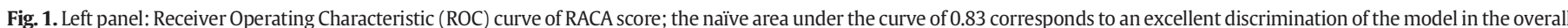

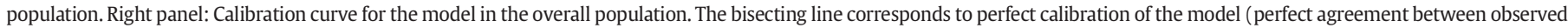

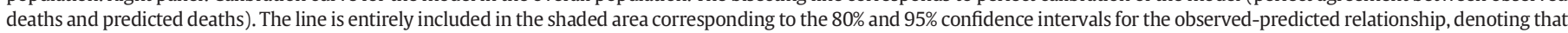
the model is well calibrated (there is neither over nor underestimation of the mortality). 


\begin{tabular}{|c|c|c|c|}
\hline $\begin{array}{c}\text { UB-ROSC } \\
\text { SCORE }\end{array}$ & $\begin{array}{c}\text { prob of } \\
\text { survival to } \\
\text { hosp adm }\end{array}$ & $\begin{array}{c}95 \% \text { lower } \\
\text { confidence } \\
\text { limit }\end{array}$ & $\begin{array}{c}95 \% \text { upper } \\
\text { confidence } \\
\text { limit }\end{array}$ \\
\hline-50 & 0.01 & 0.00 & 0.01 \\
\hline-45 & 0.01 & 0.01 & 0.02 \\
\hline-40 & 0.02 & 0.01 & 0.03 \\
\hline-35 & 0.03 & 0.02 & 0.05 \\
\hline-30 & 0.05 & 0.03 & 0.08 \\
\hline-25 & 0.08 & 0.04 & 0.12 \\
\hline-20 & 0.12 & 0.07 & 0.18 \\
\hline-15 & 0.18 & 0.11 & 0.26 \\
\hline-10 & 0.25 & 0.16 & 0.35 \\
\hline-5 & 0.35 & 0.24 & 0.47 \\
\hline 0 & 0.49 & 0.37 & 0.62 \\
\hline 5 & 0.64 & 0.51 & 0.75 \\
\hline 10 & 0.72 & 0.61 & 0.82 \\
\hline 15 & 0.80 & 0.70 & 0.88 \\
\hline 20 & 0.86 & 0.79 & 0.92 \\
\hline 30 & 0.94 & 0.90 & 0.97 \\
\hline 35 & 0.97 & 0.94 & 0.99 \\
\hline 40 & 0.98 & 0.96 & 0.99 \\
\hline 45 & 0.99 & 0.98 & 0.99 \\
\hline & & & \\
\hline
\end{tabular}

Nomogram to get the probability of survival to hospital admission given UB-ROSC Score (Random effect model)

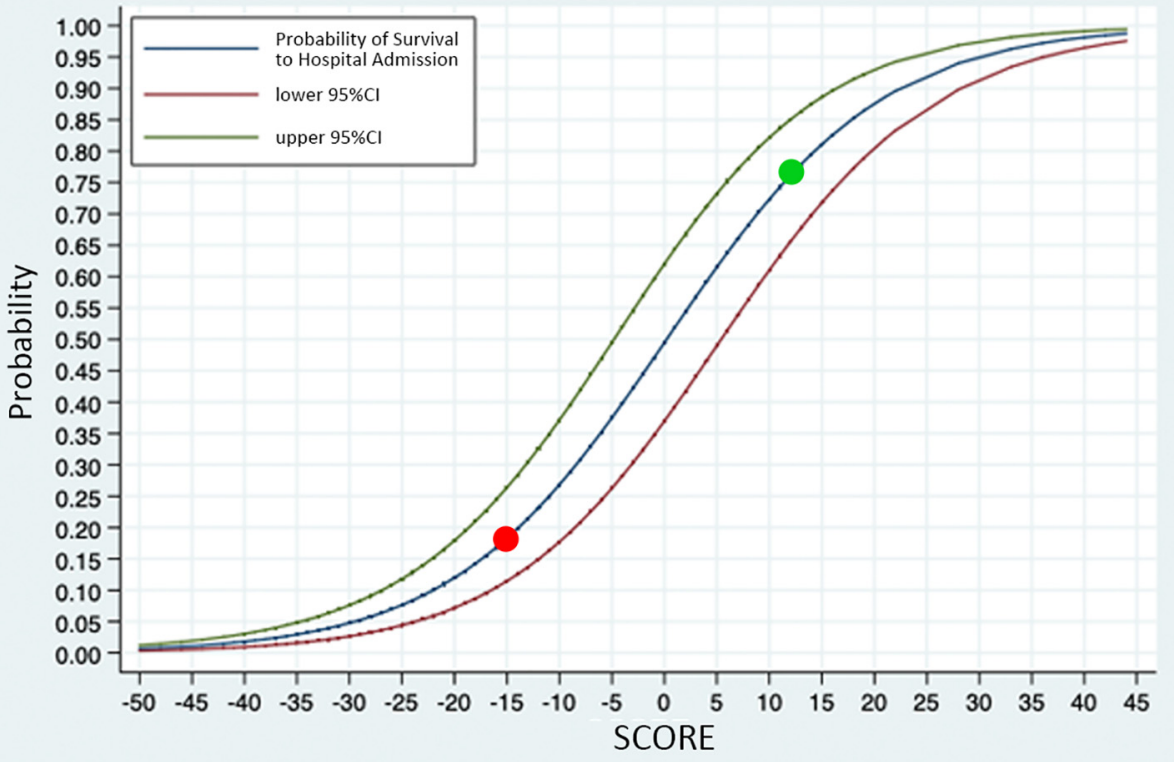

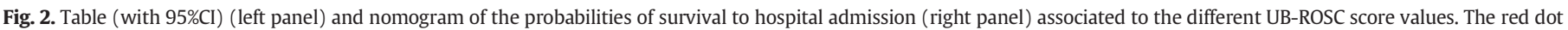

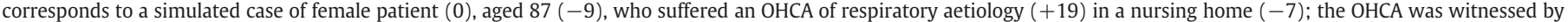

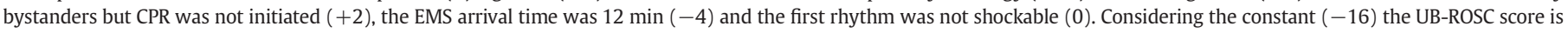

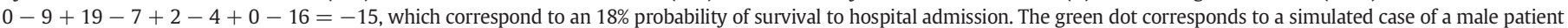

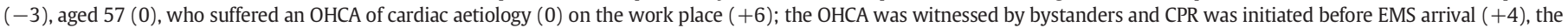

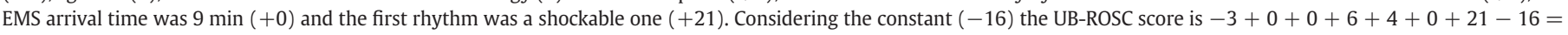

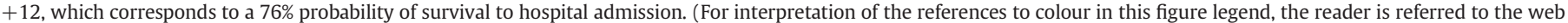
version of this article.)

and 'survival after 1 year' are clinically more relevant, but are also affected by the type, quality, and extent of post-resuscitation care that is often not standardized, and may therefore not be appropriate as a primary endpoint for scoring systems that are mainly based on preclinical data collection. Furthermore, the ACLS score may require frequent adjustments and repeated reassessments when in-hospital management strategies change, e.g. temperature management recommendation [28], percutaneous revascularization in the setting of acute myocardial infarction [29], etc., or novel approaches become available. In contrast, the UB-ROSC score considers a very well-defined and critical phase of an OHCA, i.e. the pre-hospital phase. Achieving a sustained ROSC is already a major step toward good cardio-circulatory and possibly neurological outcome.

As the RACA score, also the UB-ROSC score considers resuscitation success in the pre-hospital phase of resuscitation. The RACA score predicted endpoint is ROSC in field, defined as a palpable pulse for $\geq 20 \mathrm{~s}$. On the other hand, UB-ROSC considers only sustained ROSC as outcome allowing admission of the patient to the hospital as a clinically more relevant endpoint.

The ACLS score considered four key variables for predicting discharge alive following cardiac arrest: arrest witnessed (A), cardiac rhythm (C), lay bystander cardiopulmonary resuscitation (L) and response time of paramedic unit (speed, S). In a similar manner, UBROSC included prognostic variables readily available, but increased the number of prognostic variables including variables that, over the last 2 decades, have been consistently demonstrated to be associated with outcome [17-23]. These include patients' age and sex, aetiology of cardiac arrest, OHCA location. Notably, all variables considered in the UBROSC score are part of the uniform and modern reporting of data from OHCA developed as the Utstein Style [13]. Most of the prognostic variables included in the UB-ROSC score are similar but not identical as RACA score since this latter does not use Utstein Style variables, for both OHCA aetiology and location, thus requiring off-line adaptation.
In addition to being easier to apply, compared to RACA, the UB-ROSC performed much better. The discrimination power of UB-ROSC score was as high as 0.82 after internal and 0.77 after external validation, thus, markedly greater than the 0.71 value reported in the original publication by Gräsner et al. [23] or found in recent external validations, including our own [30-32]. Indeed, using this same data set, we found an AUC of 0.76 for the RACA score [30].

To allow an easy and simple calculation of the score by EMS personnel during a mission, a free mobile application for iOs system "UB-ROSC score" has been developed; the score is also calculable at http://www. sanmatteo.org/site/home/ub-rosc-score.html.

\subsection{Limitations}

Our study has some limitations. A first limitation is related to classification of OHCA aetiology, which was not based on the latest Utstein revision released in 2015. Since the study period started on January 1st, 2015 , our registries could not be aligned with that revision. Therefore, to limit recording errors, we maintained the 2004 categorization for the entire cohort. Although the model maintained a good performance both after internal validation on the 2015-2017 cohort and external validation on the 2018 cohort, further validation in independent cohorts is warranted for confirmation. The transferability of our results to other EMS organization that do not include a physician-staffed rescue team may be considered another limitation. Although validation in different geographical realties is highly needed, it is unlikely that the ROSC probability is lower when paramedics alone are performing advanced CPR whereas time to hospital transportation is possibly related to prehospital survival. Finally, as stated in the methods, none of the 2 EMS systems has ever applied a decision algorithm for resuscitation termination. This decision has always been left to the intervening physician discretion. For this reason, it cannot be ruled out that the discrimination ability of our score has been affected by a bias of self-fulfilling prophecy. 
However, UB-ROSC considered a derivation and validation cohort in which patient's clinical characteristics and most likely, physician decision for resuscitation termination, were similar thus, possibly mitigating this issue.

\section{Conclusions}

The UB-ROSC score is a novel score to be used in-field and predicts the probability of survival to hospital admission of OHCA victims. UBROSC is not a score to withhold resuscitation. UB-ROSC shall help in setting realistic expectations about sustained ROSC achievement during resuscitation manoeuvres, an important target for paramedics, rescue teams and even more so for family members.

\section{Acknowledgement}

We thank all the investigators of the Pavia CARe and TiReCa Registry, the EMS personnel and all the people involved in the resuscitation network of our regions.

\section{Declaration of competing interest}

The authors report no conflicts of interest related specifically to this manuscript.

\section{Appendix A. Supplementary data}

Supplementary data to this article can be found online at https://doi. org/10.1016/j.ijcard.2020.01.032.

\section{References}

[1] S.G. Priori, E. Aliot, C. Blomstrom-Lundqvist, et al., Task force on sudden cardiac death of the European Society of Cardiology, Eur. Heart J. 22 (2001 Aug) 1374-1450.

[2] F. Kette, R. Sbrojavacca, G. Rellini, et al., Epidemiology and survival rate of out-ofhospital cardiac arrest in north-east Italy: The F.A.C.S. study. Friuli Venezia Giulia Cardiac Arrest Cooperative Study, Resuscitation. 36 (1998 Mar) 153-159.

[3] J.T. Gräsner, R. Lefering, R.W. Koster, et al., EuReCa ONE Collaborators, EuReCa ONE27 Nations, ONE Europe, ONE Registry: a prospective one month analysis of out-ofhospital cardiac arrest outcomes in 27 countries in Europe, Resuscitation. 105 (2016) 188-195.

[4] K. Hayakawa, O. Tasaki, T. Hamasaki, et al., Prognostic indicators and outcome prediction model for patients with return of spontaneous circulation from cardiopulmonary arrest: the Utstein Osaka Project, Resuscitation. 82 (2011 Jul) 874-880.

[5] T.D. Valenzuela, D.J. Roe, S. Cretin, D.W. Spaite, M.P. Larsen, Estimating effectiveness of cardiac arrest interventions: a logistic regression survival model, Circulation 96 (1997) 3308-3313.

[6] E. Baldi, E. Contri, R. Burkart, D. Bywater, M. Duschl, The three dimension model of the out-of-hospital cardiac arrest, Resuscitation. 138 (2019) 44-45.

[7] L.J. Morrison, L.M. Visentin, A. Kiss, TOR Investigators, et al., Validation of a rule for termination of resuscitation in out-of-hospital cardiac arrest, N. Engl. J. Med. 355 (2006 Aug 3) 478-487.

[8] L.J. Morrison, P.R. Verbeek, C. Zhan, A. Kiss, K.S. Allan, Validation of a universal prehospital termination of resuscitation clinical prediction rule for advanced and basic life support providers, Resuscitation. 80 (2009 Mar) 324-328.

[9] Y. Goto, T. Maeda, Y.N. Goto, Termination-of-resuscitation rule for emergency department physicians treating out-of-hospital cardiac arrest patients: an observational cohort study, Crit. Care 17 (2013 Oct 13) R235.

[10] K. Shibahashi, K. Sugiyama, Y. Hamabe, A potential termination of resuscitation rule for EMS to implement in the field for out-of-hospital cardiac arrest: an observational cohort study, Resuscitation. 130 (2018 Sep) 28-32.

[11] L.L. Bossaert, G.D. Perkins, H. Askitopoulou, et al., Ethics of Resuscitation and End-oflife Decisions Section Collaborators, European Resuscitation Council Guidelines for Resuscitation 2015: Section 11. The ethics of resuscitation and end-of-life decisions, Resuscitation. 95 (2015 Oct) 302-311.

[12] I. Jacobs, V. Nadkarni, J. Bahr, International Liaison Committee on Resuscitation, American Heart Association, European Resuscitation Council, Australian Resuscitation Council, New Zealand Resuscitation Council, Heart and Stroke Foundation of Canada, InterAmerican Heart Foundation, Resuscitation Councils of Southern Africa, ILCOR Task Force on Cardiac Arrest and Cardiopulmonary Resuscitation
Outcomes, et al., Cardiac arrest and cardiopulmonary resuscitation outcome reports: update and simplification of the Utstein templates for resuscitation registries: a statement for healthcare professionals from a task force of the International Liaison Committee on Resuscitation (American Heart Association, European Resuscitation Council, Australian Resuscitation Council, New Zealand Resuscitation Council, Heart and Stroke Foundation of Canada, InterAmerican Heart Foundation, Resuscitation Councils of Southern Africa), Circulation. 110 (2004 Nov 23) 3385-3397.

[13] G.D. Perkins, I.G. Jacobs, V.M. Nadkarni, et al., Utstein Collaborators, Cardiac arrest and cardiopulmonary resuscitation outcome reports: update of the Utstein Resuscitation Registry Templates for Out-of-Hospital Cardiac Arrest: a statement for healthcare professionals from a task force of the International Liaison Committee on Resuscitation (American Heart Association, European Resuscitation Council, Australian and New Zealand Council on Resuscitation, Heart and Stroke Foundation of Canada, InterAmerican Heart Foundation, Resuscitation Council of Southern Africa, Resuscitation Council of Asia); and the American Heart Association Emergency Cardiovascular Care Committee and the Council on Cardiopulmonary, Critical Care, Perioperative and Resuscitation, Circulation. 132 (2015) 1286-1300.

[14] R. Mauri, R. Burkart, C. Benvenuti, et al., Better management of out-of-hospital cardiac arrest increases survival rate and improves neurological outcome in the Swiss Canton Ticino, Europace. 18 (2016 Mar) 398-404.

[15] S. Savastano, S. De Servi, L. Oltrona Visconti, M. Raimondi, All the Pavia CARE researchers, The Pavia Cardiac Arrest REgistry - Pavia CARE. It's time for cardiologists to be engaged in cardiac arrest, Int. J. Cardiol. 185 (2015) 93-94.

[16] M.L. Caputo, S. Muschietti, R. Burkart, et al., Lay persons alerted by mobile application system initiate earlier cardio-pulmonary resuscitation: a comparison with SMS-based system notification, Resuscitation. 114 (2017 May) 73-78.

[17] J. Herlitz, J. Bahr, M. Fischer, M. Kuisma, K. Lexow, G. Thorgeirsson, Resuscitation in Europe: a tale of five European regions, Resuscitation 41 (1999) 121-131.

[18] Breckwoldt J, Schloesser S, Arntz HR. Perceptions of collapse and assessment of cardiac arrest by bystanders of out-of-hospital cardiac arrest (OOHCA). Resuscitation2009;80:1108-1113.

[19] J. Herlitz, J. Engdahl, L. Svensson, M. Young, K.A. Angquist, S. Holmberg, Can we define patients with no chance of survival after out-of-hospital cardiac arrest? Heart 90 (2004) 1114-1118.

[20] J. Herlitz, J. Engdahl, L. Svensson, M. Young, K.A. Angquist, S. Holmberg, Decrease in the occurrence of ventricular fibrillation as the initially observed arrhythmia after out-of-hospital cardiac arrest during 11 years in Sweden, Resuscitation 60 (2004) $283-290$.

[21] R.B. Vukmir, Witnessed arrest, but not delayed bystander cardiopulmonary resuscitation improves prehospital cardiac arrest survival, Emerg. Med. J. 21 (2004) 370-373.

[22] R.A. Waalewijn, J.G. Tijssen, R.W. Koster, Bystander initiated actions in out-ofhospital cardiopulmonary resuscitation: results from the Amsterdam Resuscitation Study (ARRESUST), Resuscitation 50 (2001) 273-279.

[23] J.T. Gräsner, P. Meybohm, R. Lefering, German Resuscitation Registry Study Group et al., ROSC after cardiac arrest-the RACA score to predict outcome after out-ofhospital cardiac arrest, Eur. Heart J. 32 (2011 Jul) 1649-1656.

[24] M.E. Ong, J. Jaffey, I. Stiell, L. Nesbitt, OPALS Study Group, Comparison of termination-of-resuscitation guidelines for basic life support: defibrillator providers in out-of-hospital cardiac arrest, Ann. Emerg. Med. 47 (2006) 337-343.

[25] C. Sasson, A.J. Hegg, M. Macy, A. Park, A. Kellermann, B. McNally, CARES Surveillance Group, Prehospital termination of resuscitation in cases of refractory out-of-hospital cardiac arrest, JAMA. 300 (2008) 1432-1438, https://doi.org/10.1001/jama.300.12. 1432.

[26] M. Eisenberg, A. Hallstrom, L. Bergner, The ACLS score. Predicting survival from outof-hospital cardiac arrest, JAMA 246 (1981) 50-52.

[27] R.O. Cummins, J.P. Ornato, W.H. Thies, P.E. Pepe, Improving survival from sudden cardiac arrest: the "chain of survival" concept. A statement for health professionals from the Advanced Cardiac Life Support Subcommittee and the Emergency Cardiac Care Committee, American Heart Association, Circulation. 83 (1991) 1832-1847.

[28] N. Nielsen, J. Wetterslev, T. Cronberg, TTM Trial Investigators, et al., Targeted temperature management at $33{ }^{\circ} \mathrm{C}$ versus $36{ }^{\circ} \mathrm{C}$ after cardiac arrest, N. Engl. J. Med. 369 (2013) 2197-2206, https://doi.org/10.1056/NEJMoa1310519.

[29] P. Garot, T. Lefevre, H. Eltchaninoff, et al., Six-month outcome of emergency percutaneous coronary intervention in resuscitated patients after cardiac arrest complicating ST-elevation myocardial infarction, Circulation 115 (11) (2007) 1354-1362.

[30] M.L. Caputo, E. Baldi, S. Savastano, et al., Validation of the return of spontaneous circulation after cardiac arrest (RACA) score in two different national territories, Resuscitation 134 (2018 Nov 14) 62-68, https://doi.org/10.1016/j.resuscitation.2018.11. 012.

[31] P. Kupari, M. Skrifvars, M. Kuisma, External validation of the ROSC after cardiac arrest (RACA) score in a physician staffed emergency medical service system, Scand J Trauma Resusc Emerg Med. 25 (2017 Mar 29) 34.

[32] J.C. Schewe, J. Kappler, U. Heister, et al., Outcome of out-of-hospital cardiac arrest over a period of 15 years in comparison to the RACA score in a physician staffed urban emergency medical service in Germany, Resuscitation. 96 (2015 Nov) $232-238$. 